4 Tekant G, Eroglu E, Erdogan E et al. Corrosive injury-induced gastric outlet obstruction: a changing spectrum of agents and treatment. J. Pediatr. Surg. 2001; 36: 1004-7.

5 Edwards MJ, Kollenberg SJ, Brandt ML et al. Surgery for peptic ulcer disease in children in the post-histamine-blocker era. J. Pediatr. Surg. 2005; 40: 850-4.

6 Azarow K, Kim P, Ein S. A 45-year experience with surgical treatment of peptic ulcer disease in children. J. Pediatr. Surg. 1996; 31: $750-3$.

7 Lin J, Lee ZF, Yen YC et al. Pneumatic dilation in treatment of late-onset primary gastric outlet obstruction in childhood. J. Pediatr. Surg. 2007; 42: e1-e4.
8 Chan KL, Saing H. Balloon catheter dilatation of peptic pyloric stenosis in children. J. Pediatr. Gastroenterol. Nutr. 1994; 18: 465-8.

9 Misra SP, Dwivedi M. Long-term follow-up of patients undergoing balloon dilation for benign pyloric stenosis. Endoscopy 1996; 28: $552-4$.

10 Israel DM, Mahdt G, Hassall E. Pyloric balloon dilation for delayed gastric emptying in children. Can. J. Gastroenterol. 2001; 15: 723-7.

\title{
Pediatric case of mesalazine-induced interstitial nephritis with literature review
}

\author{
Maridine L. Co and Emmanuel C. Gorospe \\ Department of Pediatrics, Children's Hospital of Michigan, Wayne State University School of Medicine, Detroit, Michigan, \\ USA
}

\begin{abstract}
We present the case of a 14-year-old boy with ulcerative colitis who was diagnosed with mesalazine-induced interstitial nephritis (M-IIN). Improvement in renal function occurred with discontinuation of mesalazine and corticosteroid therapy. We systematically searched the literature for pediatric cases of M-IIN. There were eight cases. Majority of the cases were boys $(75 \%)$ with ulcerative colitis $(75 \%)$. Average duration of mesalazine use prior to the diagnosis of interstitial nephritis was $24 \pm 18$ months. The median dose was $1.5 \mathrm{~g} /$ day. M-IIN appears to be an idiosyncratic reaction without any relation to dose or duration of mesalazine use. Although there are no guidelines to recommend routine surveillance of renal function, monitoring of serum creatinine in patients on mesalazine remains an inexpensive and non-invasive test that may lead to early detection and treatment of renal injury.
\end{abstract}

Key words inflammatory bowel disease, interstitial nephritis, mesalazine.

Mesalazine has been used as first-line therapy for mild to moderate inflammatory bowel disease (IBD). It is molecularly similar to salicylates and phenacetin. Its mechanism of action in controlling IBD remains unclear. Although safe in general, mesalazine use has been associated with fever, watery diarrhea, thrombocytopenia, pancreatitis, pneumonitis, and pericarditis. ${ }^{1}$ Among these adverse drug-related events, interstitial nephritis is a rare but serious complication of long-term mesalazine use. The first reported association of interstitial nephritis with 5-aminosalicylates (5-ASA) was in 1992. ${ }^{2}$ Most reported cases of mesalazine-associated interstitial nephritis are in adults. We present an interesting case of mesalazine-induced interstitial nephritis (M-IIN) in an adolescent patient with ulcerative colitis. We provide further information on this rare condition by

Correspondence: Maridine L. Co, MD, CNSC, Detroit Medical Center, 3737 Beaubien Street, Detroit, MI 48201, USA. Email: mco@dmc.org

Received 13 December 2011; revised 5 February 2012; accepted 19 September 2012.

doi: 10.1111/j.1442-200X.2012.03745.x presenting a systematic review of the few published case reports of pediatric M-IIN in the literature.

\section{Case Report}

A 14-year-old boy with a 5-year history of ulcerative colitis presented with a 2-week history of progressive fatigue, followed by nausea and vague abdominal pain 5 days prior to admission. His ulcerative colitis had been on clinical remission while on mesalazine $(1.5 \mathrm{~g} /$ day $)$ for the past 3 years. Aside from ulcerative colitis, the patient had no other medical history, no prior use of non-steroidal anti-inflammatory drugs, or history of renal insufficiency. He reported having frothy non-foul-smelling urine recently but denied dysuria, hematuria, or changes in his urinary frequency. His vital signs were as follows: temperature $36.8^{\circ} \mathrm{C}$, blood pressure $90 / 60 \mathrm{~mm} \mathrm{Hg}$, heart rate 98 b.p.m., and respiratory rate 20 breaths/min. He had no focal abdominal tenderness, rebound, guarding, flank, or suprapubic pain. He had grade 1 bipedal edema but no evidence of periorbital swelling or anasarca. The patient was found to be in renal failure with the following laboratory parameters: serum creatinine $11.9 \mathrm{mg} / \mathrm{dL}$, 


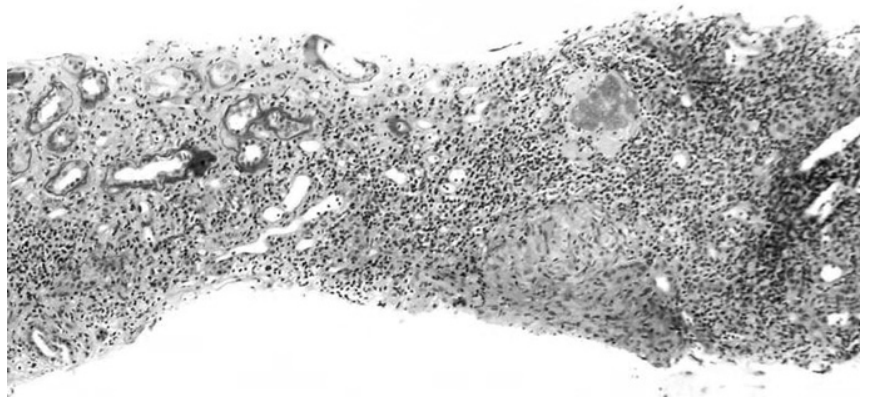

Fig. 1 Microscopic findings in the renal biopsy: mononuclear infiltration (lymphocytes) with scattered eosinophils in the interstitium (Periodic acid-Schiff stain).

urea $63 \mathrm{mg} / \mathrm{dL}$, potassium of $4.8 \mathrm{mg} / \mathrm{dL}$, and phosphorus of $9 \mathrm{mg} / \mathrm{dL}$. Hemoglobin was $10.3 \mathrm{~g} / \mathrm{dL}$, white cell count of $14.8 \times 10^{3} / \mathrm{mm}^{3}$, absolute eosinophil count was $0.4 \times 10^{3} / \mathrm{mm}^{3}$, and platelets of $189 \times 10^{3} / \mathrm{mm}^{3}$. Urinalysis showed $40 / \mathrm{hpf}$ leukocytes without any eosinophiluria, hematuria or bacteriuria. Proteinuria of $600 \mathrm{mg}$ was detected in a 24-h urine collection. The patient's blood cultures were negative for both aerobic and anaerobic bacteria. Screening serologic tests for HIV, viral hepatitis, herpes simplex virus, cytomegalovirus, and rickettsial diseases were all negative. Mycobacterium tuberculosis infection was ruled out by quantiferon testing. Rheumatologic screening with anti-nuclear and double-stranded DNA autoantibodies was negative. The ultrasound of the kidneys did not show evidence of cortical atrophy, hydronephrosis, or nephrolithiasis. During his initial hospital course, the patient received aggressive fluid resuscitation and electrolyte management, resulting in slight improvement of his serum creatinine to $9 \mathrm{mg} / \mathrm{dL}$ in 2 days. He underwent renal biopsy, which showed active mononuclear infiltration with scattered eosinophils, consistent with interstitial nephritis (Fig. 1). Given the history of long-term mesalazine use, the histopathologic findings were consistent with mesalazine-associated interstitial nephritis. Mesalazine was discontinued and the patient was started on i.v. methylprednisolone ( $1 \mathrm{mg} / \mathrm{kg} / \mathrm{day})$ for 3 days.
He was discharged from the hospital with improvement of his symptoms and was prescribed oral prednisone taper for 2 months. His creatinine decreased to $1.4 \mathrm{mg} / \mathrm{dL}$ in 8 weeks. In spite of discontinuing his mesalazine regimen, the patient continued to be in clinical remission in terms of his ulcerative colitis.

\section{Discussion}

We conducted a systematic search on PubMed from January 1992 to November 2011 for pediatric cases of M-IIN, confirmed by clinical, laboratory, and histopathologic criteria. We used the following search terms: "5-aminosalicylic acid", "mesalazine", "mesalamine", "renal failure", and "inflammatory bowel disease" per the Cochrane collaboration search strategy. We reviewed the bibliographies of our selected case reports for additional references. We retrieved eight pediatric cases of interstitial nephritis associated with mesalazine use for the treatment of IBD. Both authors independently extracted pertinent clinical data, summarized in Table 1 . The previously reported cases were similar to our present case. Previously published pediatric cases of M-IIN were predominantly in boys $(n=6 ; 75 \%)$, mean age was $15.4 \pm 2.4$ years old, and they had ulcerative colitis $(n=6$; $75 \%)$. The baseline renal function in all patients was normal. All patients presented with non-specific signs and symptoms, such as fever, fatigue, weight loss, abdominal pain, nausea, and diarrhea, prior to the diagnosis of interstitial nephritis. Urinalysis in all patients was remarkable for proteinuria except for two cases, which only reported white blood cells. There were only two cases that reported the presence of eosinophils in urine. ${ }^{8}$ The finding of eosinophiluria lacks sensitivity and specificity to either diagnose or rule out interstitial nephritis. ${ }^{9}$ Renal ultrasound was abnormal in four cases (50\%), revealing increased renal cortical echogenicity and decreased corticomedullary differentiation. Confirmatory renal biopsy was reported in seven out of eight patients. The case reported by Uslu et al. ${ }^{4}$ did not have histologic confirmation because of the family's refusal to consent for kidney biopsy. Nevertheless, the patient's clinical course and laboratory findings were consistent with interstitial nephritis. In cases that reported

Table 1 Published reports of M-IIN in pediatric patients

\begin{tabular}{|c|c|c|c|c|c|c|c|c|}
\hline Uslu et al. ${ }^{4}$ & $15 / F$ & UC & 0.8 & 1.1 & 1.5 & 4 & Yes & Recovered \\
\hline Arend et $a .^{2}$ & $18 / \mathrm{M}$ & $\mathrm{UC}$ & 1 & 2.5 & 1.2 & 18 & Yes & Recovered \\
\hline Skalova et al. ${ }^{5}$ & $15 / \mathrm{M}$ & UC & NR & 1.43 & 3 & 48 & Yes & Recovered \\
\hline Van Biervliet et al. ${ }^{6}$ & $11 / \mathrm{F}$ & UC & NR & 0.88 & 1.5 & 36 & Yes & Recovered \\
\hline \multirow[t]{2}{*}{ Benador et al. ${ }^{8}$} & $16 / \mathrm{M}$ & $\mathrm{CD}$ & NR & 2.5 & NR & 6 & Yes & Recovered \\
\hline & 14/M & $\mathrm{CD}$ & 0.8 & 1.4 & NR & 11 & Yes & Recovered \\
\hline Current Patient & $14 / \mathrm{M}$ & $\mathrm{UC}$ & 0.8 & 13.9 & 1.5 & 36 & Yes & Recovered \\
\hline
\end{tabular}

5-ASA, 5-aminosalicylates; CD, Crohn's disease; ESRD, end-stage renal disease; IBD, inflammatory bowel disease; M-IIN, mesalazine-induced interstitial nephritis; NR, not reported; UC, ulcerative colitis. 
mesalazine dose, the median was $1.5 \mathrm{~g} /$ day (range $1.2-3.0 \mathrm{~g} /$ day). Average duration of mesalazine use prior to the diagnosis of interstitial nephritis was $23.8 \pm 18.2$ months. There were seven cases that improved with discontinuation of mesalazine and treatment with steroids, similar to our case. There was only one case that developed end-stage renal disease, requiring hemodialysis. ${ }^{3}$

Interstitial nephritis is a rare but potentially serious complication of mesalazine use. An analysis of a prescription database in the UK from 1991 to 1998 estimated about 11 reports of interstitial nephritis for every million prescriptions of mesalazine.$^{10}$ In terms of other 5-ASA products, a retrospective analysis of the UK General Practice Research Database on adult patients did not show any difference in the risk of developing interstitial nephritis with other 5-ASA drugs, such as balsalazide and olsalazine. ${ }^{11}$ However, there are no specific studies in children comparing the incidence of interstitial nephritis with mesalamine and other 5-ASA products. To our knowledge, our systematic review is one of the few studies to report 5-ASA-associated interstitial nephritis in pediatric patients.

The mechanism of renal injury from mesalazine remains unclear. There are preclinical studies linking aminosalicylates to renal papillary necrosis but not directly with interstitial nephritis. Based on animal models and clinical experience from adult patients, M-IIN appears to be an idiosyncratic reaction without any relation to the dose given to patients. ${ }^{2}$ Renal insufficiency may also be a complication of IBD by itself. Renal involvements in IBD may include: calcium oxalate stone formation, nephrolithiasis, nephrocalcinosis, ureteric obstruction, immune complex glomerulonephritis, and secondary amyloidosis. ${ }^{12}$ In our case report, the temporal association of renal failure and recovery with the use and discontinuation of mesalazine, respectively, is indicative of a drug-induced mechanism rather than a primary IBD-associated process. In contrast, failure to respond to mesalazine discontinuation and steroid therapy are the hallmarks of IBD-associated renal dysfunction. ${ }^{12}$

In our review of the published pediatric M-IIN cases, the duration of 5-ASA use prior to the onset of acute renal injury varied from 4 to 48 weeks (Table 1). This is similar to the onset of other drug-induced interstitial nephritis where patients present with either acute or subacute onset of non-specific symptoms, such as malaise, anorexia, oliguria or nausea and vomiting. Based on the kidney biopsy results, our case was diagnosed as acute interstitial nephritis in the absence of chronic changes, such as interstitial scarring, fibrosis or tubular atrophy. Also, the onset of symptoms was only 2 weeks. Prior to this, the patient was asymptomatic while on mesalazine for 3 years. Similar to most patients with drug-induced interstitial nephritis, withdrawal of the offending drug and administration of steroids may show recovery of renal function to normal or near-normal levels within a few weeks. ${ }^{9}$

Although there are no standard guidelines to recommend routine surveillance of renal function using serum creatinine and urinalysis in IBD patients on mesalazine therapy, monitor- ing of serum creatinine remains a simple, inexpensive, and a non-invasive laboratory test which may detect and prevent progression of renal injury. Frandsen et al. ${ }^{3}$ suggested practical recommendations for measurement of serum creatinine prior to the start of mesalazine treatment, followed by monthly monitoring for the first 3 months, every 3 months for the next 9 months, and every 6 months thereafter.

\section{Conclusion}

Mesalazine-induced interstitial nephritis is an infrequent but significant adverse event that has occurred in pediatric IBD patients. Early recognition of renal insufficiency may prevent further progression to end-stage renal disease. If detected early, discontinuation of mesalazine and trial of corticosteroid therapy may facilitate recovery of renal function.

\section{Acknowledgments}

The authors declare no conflict of interest. The authors assure that no identifiable patient information is contained in this publication, for the protection of the patient's privacy.

\section{References}

1 Hanauer SB. Review article: evolving concepts in treatment and disease modification in ulcerative colitis. Aliment. Pharmacol. Ther. 2008; 27 (Suppl. 1): 15-21.

2 Arend LJ, Springate JE. Interstitial nephritis from mesalazine: case report and literature review. Pediatr. Nephrol. 2004; 19: 550-3.

3 Frandsen NE, Saugmann S, Marcussen N. Acute interstitial nephritis associated with the use of mesalazine in inflammatory bowel disease. Nephron 2002; 92: 200-2.

4 Uslu N, Demir H, Saltik-Temizel IN, Topaloglu R, Gurakan F, Yuce A. Acute tubular injury associated with mesalazine therapy in an adolescent girl with inflammatory bowel disease. Dig. Dis. Sci. 2007; 52: 2926-9.

5 Skalova S, Dedek P, Pozler O, Podhola M. Mesalazine-induced interstitial nephritis. Ren. Fail. 2009; 31: 159-61.

6 Van Biervliet S, Raes A, Vande Walle J, Van Winckel M, Robberecht E, Praet M. Mesalazine interstitial nephritis presenting as colitis ulcerosa exacerbation. Acta Gastroenterol Belg. 2006; 69: 321-2.

7 Alivanis P, Aperis G, Lambrianou F et al. Reversal of refractory sulfasalazine-related renal failure after treatment with corticosteroids. Clin. Ther. 2010; 32: 1906-10.

8 Benador N, Grimm P, Lemire J, Griswold W, Billman G, Reznik V. Interstitial nephritis in children with Crohn's disease. Clin. Pediatr. (Phila) 2000; 39: 253-4.

9 Ten RM, Torres VE, Milliner DS, Schwab TR, Holley KE, Gleich GJ. Acute interstitial nephritis: immunologic and clinical aspects. Mayo Clin. Proc. 1988; 63: 921-30.

10 Ransford RA, Langman MJ. Sulphasalazine and mesalazine: serious adverse reactions re-evaluated on the basis of suspected adverse reaction reports to the Committee on Safety of Medicines. Gut 2002; 51: 536-9.

11 Van Staa TP, Travis S, Leufkens HG, Logan RF. 5-aminosalicylic acids and the risk of renal disease: a large British epidemiologic study. Gastroenterology 2004; 126: 1733-9.

12 Izzedine H, Simon J, Piette AM et al. Primary chronic interstitial nephritis in Crohn's disease. Gastroenterology 2002; 123: 143640 . 